\title{
Redistribution of the Lamin B1 genomic binding profile affects rearrangement of heterochromatic domains and SAHF formation during senescence
}

\author{
Mahito Sadaie, ${ }^{1,6,7}$ Rafik Salama, ${ }^{1,6}$ Thomas Carroll, ${ }^{1}$ Kosuke Tomimatsu, ${ }^{1,2}$ Tamir Chandra, ${ }^{1,8}$ \\ Andrew R.J. Young, ${ }^{1}$ Masako Narita, ${ }^{1}$ Pedro A. Pérez-Mancera, ${ }^{1}$ Dorothy C. Bennett, ${ }^{3}$ Heung Chong, ${ }^{4}$ \\ Hiroshi Kimura, ${ }^{5}$ and Masashi Narita ${ }^{1,9}$ \\ ${ }^{1}$ Cancer Research UK Cambridge Institute, Li Ka Shing Centre, University of Cambridge, Cambridge CB2 0RE, United Kingdom; \\ ${ }^{2}$ Research Centre for Bioscience and Technology, Tottori University, Tottori 683-8503, Japan; ${ }^{3}$ Biomedical Sciences Research \\ Centre, St. George's, University of London, London SW17 0RE, United Kingdom; ${ }^{4}$ Cellular Pathology, Division of Biomedical \\ Sciences, St. George's, University of London, London SW17 0RE, United Kingdom; ${ }^{5}$ Graduate School of Frontier Biosciences, \\ Osaka University, Osaka 565-0871, Japan
}

Senescence is a stress-responsive form of stable cell cycle exit. Senescent cells have a distinct gene expression profile, which is often accompanied by the spatial redistribution of heterochromatin into senescence-associated heterochromatic foci (SAHFs). Studying a key component of the nuclear lamina lamin B1 (LMNB1), we report dynamic alterations in its genomic profile and their implications for SAHF formation and gene regulation during senescence. Genome-wide mapping reveals that LMNB1 is depleted during senescence, preferentially from the central regions of lamina-associated domains (LADs), which are enriched for Lys9 trimethylation on histone $\mathrm{H} 3$ (H3K9me3). LMNB1 knockdown facilitates the spatial relocalization of perinuclear H3K9me3-positive heterochromatin, thus promoting SAHF formation, which could be inhibited by ectopic LMNB1 expression. Furthermore, despite the global reduction in LMNB1 protein levels, LMNB1 binding increases during senescence in a small subset of gene-rich regions where $\mathrm{H} 3 \mathrm{~K} 27 \mathrm{me} 3$ also increases and gene expression becomes repressed. These results suggest that LMNB1 may contribute to senescence in at least two ways due to its uneven genome-wide redistribution: first, through the spatial reorganization of chromatin and, second, through gene repression.

[Keywords: Lamin B1; senescence; epigenetics]

Supplemental material is available for this article.

Received March 6, 2013; revised version accepted July 22, 2013.

Cellular senescence is a state of stable cell cycle exit triggered by cellular stress, including genotoxic, oxidative, and oncogenic stress. Senescence is characterized by the activation of tumor suppressor proteins, including p53 and $\mathrm{Rb}$, and limits cellular immortalization in culture. These tumor-suppressive hallmarks are reinforced by the paradoxical induction of senescence observed upon the activation or loss of certain oncogenes or tumor suppressors in both cultured cells and premalignant tumors

\footnotetext{
${ }^{6}$ These authors contributed equally to this work. Present addresses: ${ }^{7}$ Graduate School of Biostudies, Kyoto University, Kyoto 606-8501, Japan; ${ }^{8}$ Laboratory of Developmental Genetics and Imprinting, The Babraham Institute, Cambridge CB22 3AT, UK. ${ }^{9}$ Corresponding author

E-mail masashi.narita@cruk.cam.ac.uk

Article is online at http://www.genesdev.org/cgi/doi/10.1101/gad.217281.113. Freely available online through the Genes \& Development Open Access option.
}

(Serrano et al. 1997; Collado and Serrano 2006). Senescence has also been implicated in aging: Cells derived from premature aging syndrome patients become senescent more readily in culture compared with young or healthy individuals (McClintock et al. 2006). Age-dependent accumulation of senescent cells in progenitor compartments has also been observed in various tissues, limiting the regenerative capacity of those organs during aging (Sharpless and DePinho 2007).

Senescent cells exhibit dynamic gene expression changes, including some specific features such as the up-regulation of p16INK4A and components of senescence-associated secretory phenotype (SASP) as well as the stable repression of proliferation-related genes (Shelton et al. 1999). In addition, senescence is often accompanied by changes in chromatin structure, forming senescence-associated heterochromatic foci (SAHFs) (Narita et al. 2003). Through the study of senescence induced by the ectopic expres- 
sion of oncogenic Ras in human fibroblasts, a number of functional and physical components of the process of SAHF formation have been identified (Chan et al. 2005; Zhang et al. 2005; Funayama et al. 2006; Narita et al. 2006; Ye et al. 2007). SAHFs are highly organized structures, where Lys9 trimethylation on histone H3 (H3K9me3; a constitutive heterochromatin marker) forms the core, which is surrounded by a layer of H3K27me3 (a facultative heterochromatin marker). These repressive layers are clearly separated from the outer transcriptionally active layer, supporting the idea that SAHF formation may contribute to gene expression profile stability for both active and repressive genes, although the direct relationship between SAHFs and gene regulation is still elusive (Chandra and Narita 2013). Despite the striking structural alteration in chromatin, the global landscapes of the repressive histone marks are highly static during SAHF formation, with only localized alterations in some genic regions, thus suggesting that SAHFs are formed through a spatial repositioning of repressively marked chromatin (Chandra et al. 2012).

The nuclear lamina is a filamentous structure, forming a scaffold underneath the inner nuclear membrane. In addition to its role in maintaining nuclear structural integrity, it has been implicated in the nuclear positioning of chromatin and transcription regulation (Dechat et al. 2010; Kind and Van Steensel 2010; Peric-Hupkes et al. 2010; Dittmer and Misteli 2011; Burke and Stewart 2012). The major structural components of the lamina in mammals are the intermediate filament proteins Lamin A/C (LMNA/C), LMNB1, and LMNB2. Genome-wide mapping of LMNB1 identified large lamina-associated domains (LADs), which are devoid of active histone marks and enriched for repressive marks (Pickersgill et al. 2006; Guelen et al. 2008). LADs are relatively gene-poor, and those genes included in LADs are generally silenced in both humans and Drosophila. During mouse embryonic stem cell (ESC) differentiation, the overall LAD pattern remains largely static. However, more localized changes in lamina-chromatin association are also observed, and their correlation with gene expression reinforces the idea that LADs provide a transcriptionally repressive environment (Peric-Hupkes et al. 2010).

Recent reports have shown that the global LMNB1 level is altered during senescence (Shimi et al. 2011; Barascu et al. 2012; Freund et al. 2012; Dreesen et al. 2013). However, it is unclear how the LMNB1 alterations affect the dynamic alterations seen in chromatin structure and the gene expression profile during senescence. Here we show that the alterations in LMNB1 in senescent human diploid fibroblasts (HDFs) are far from uniform along the genome. Despite the global down-regulation in LMNB1, LMNB1 binding is reduced mainly in H3K9me3enriched regions, and this reduction is correlated with the spatial repositioning of $\mathrm{H} 3 \mathrm{~K} 9 \mathrm{me} 3$-enriched chromatin but not with gene expression changes during senescence. Furthermore, we also found de novo gains in LMNB1 binding in small sections of the genome, which are correlated with increased $\mathrm{H} 3 \mathrm{~K} 27 \mathrm{me} 3$ and gene repression. Our data suggest that the alterations in LMNB1-genome bind- ing have different roles between regions with different chromatin states.

\section{Results \\ LMNB1 genomic binding is redistributed during senescence}

We first examined the senescence-associated alteration in the LMNB1 level using IMR90 HDFs. Consistent with previous reports (Shimi et al. 2011; Freund et al. 2012; Dreesen et al. 2013), total LMNB1 protein levels were down-regulated during oncogenic H-Ras-induced senescence (RIS) and replicative exhaustion senescence but not in quiescent cells (induced by serum starvation) or cells immortalized through adenoviral oncoprotein E1A expression (Fig. 1A; Supplemental Fig. S1A,B). The chromatinbound LMNB1 level was also reduced in RIS cells (Supplemental Fig. S1C).

To analyze how the genome-wide LMNB1 DNA binding profile changes during senescence, we performed three replicates of LMNB1 chromatin immunoprecipitation (ChIP) followed by genome-wide parallel sequencing (ChIP-seq; mean Spearman correlation coefficient $\left.r_{s}=0.9\right)$ in growing and RIS IMR90 cells. Since the global level of LMNB1 protein in RIS was substantially reduced, the LMNB1 ChIP-seq libraries generated from RIS cells suffered from lower complexity, which was taken into account through intercondition and intracondition normalization for all read-based analyses (Supplemental Material; Supplemental Fig. S2). The similarity between our ChIP-seq results (growing cells) and the LADs identified in different HDFs by the DamID (DNA adenine methyltransferase identification) technique using a DamLMNB1 fusion protein (Guelen et al. 2008) was high $\left(r_{s}=\right.$ $0.7)$, and $>80 \%$ of DamID-defined LADs were detected by ChIP-seq (Supplemental Table S1; Supplemental Fig. S3, see the legends for details). Consistent with the global down-regulation in the LMNB1 protein level, LMNB1binding events were reduced overall during RIS (Fig. 1B,C). Nevertheless, we identified a substantial number of LADs in RIS cells (Supplemental Table S1). Indeed, in some regions, LMNB1 binding was even increased. The increase of LMNB1 binding was confirmed for several regions by independent ChIP-qPCR experiments, thereby supporting our normalization method for the LMNB1 ChIP-seq data across the two conditions (Supplemental Fig. S4). Thus, despite the global reduction in LMNB1 protein levels, the alterations in the LMNB1 binding profile during RIS were not uniform across the genome.

\section{LMNB1 is preferentially reduced from H3K9me3 regions}

To investigate the changes in the LMNB1 binding profile further, we divided the genome into six classes according to the differential LMNB1 binding between growing and RIS (Fig. 1D, see the legend for details). LMNB1-decreased regions ("Grow-only" + "RIS-down") contained a relatively low frequency of genes (Grow-only in particular), CpG islands, and promoters (Fig. 1E; Supplemental Fig. S5 
A

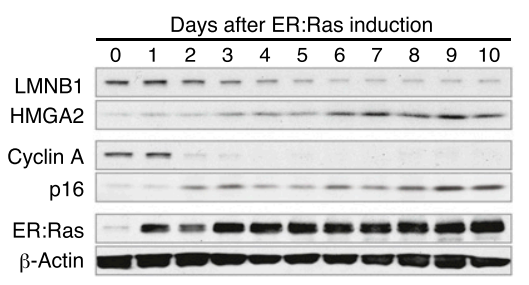

C

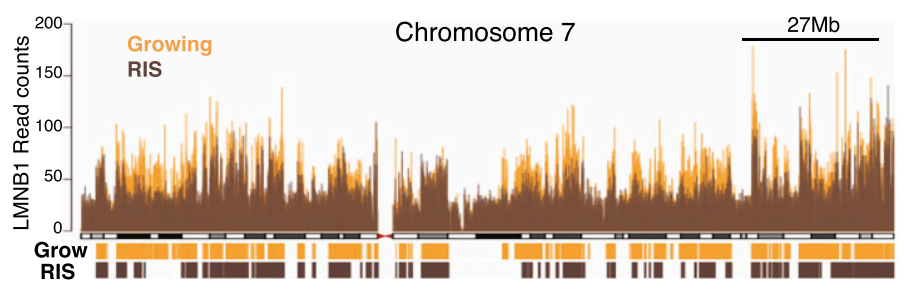

D

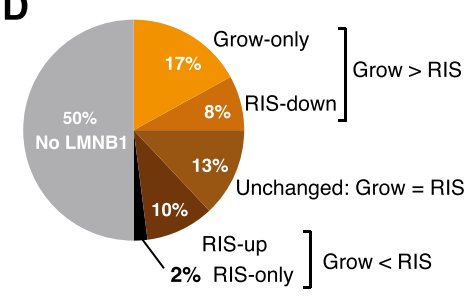

E
B
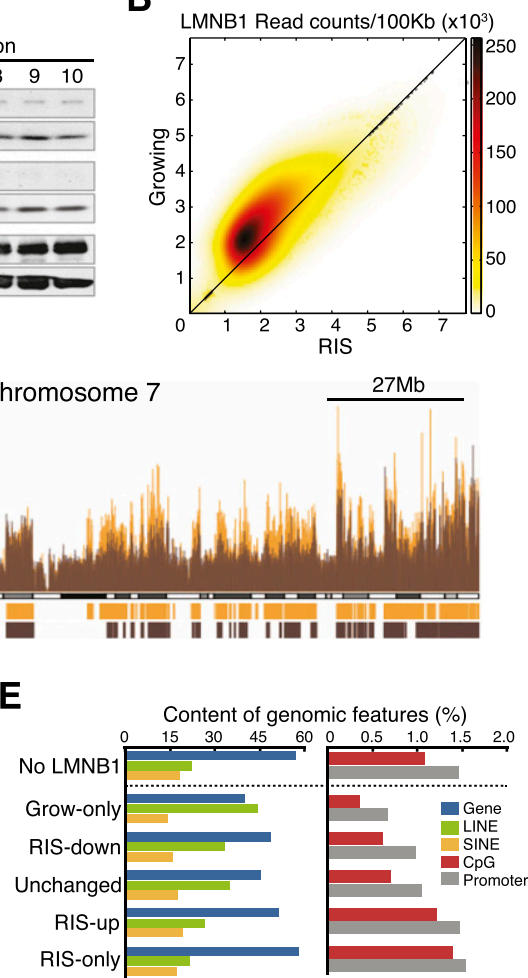

Figure 1. Uneven alterations in LMNB1 genomic profile during RIS. (A) Western blotting with the indicated antibodies for whole-cell lysates collected over 10 consecutive days after estrogen receptor (ER)-tagged H-Ras ${ }^{\mathrm{G} 12 \mathrm{~V}}$ (ER:Ras) induction by adding $100 \mathrm{nM}$ 4-hydroxytamoxifen (4-OHT) in IMR90 cells. $(B)$ A smoothhist2D plot showing the global reduction of LMNB1 during RIS. Normalized ChIPseq counts of LMNB1 in 100,000-segmented regions in growing versus RIS cells are plotted (see the Materials and Methods for normalization). Only fragments that are LMNB1-positive in either condition were included. $(C)$ A representative wholechromosome browser snapshot overlap of normalized ChIP-seq reads from growing and RIS IMR90 conditions. $(D)$ Pie chart describing six nonoverlapping classes of genomic regions based on differential binding events of LMNB1 between growing and RIS. Numbers represent percentage of the genome in each class. ("RIS-only" or "Grow-only") LMNB1 is positive only in one condition; ("RIS-up" or "RISdown") LMNB1 is positive in both conditions, and its levels are significantly up-regulated or downregulated, respectively, in RIS cells; ("Unchanged") LMNB1 is positive in both conditions with no significant differences. (E) Genomic features of each class as defined in $D$. The features are represented as a percentage of total base pairs in each class. (Gene) Protein-coding gene bodies; (LINE) long interspersed nuclear element; (SINE) short interspersed nuclear element; (CpG) CpG islands; (Promoter) -1.5 to +0.5 $\mathrm{kb}$ of transcription start sites.

shows three representative examples of genome browser shots of LMNB1 ChIP-seq from "RIS-only" regions that overlap with genes). Consistently, chromosome-wide analyses revealed that the extent of LMNB1 reduction varied between chromosomes with the more prominent reductions in gene-poor chromosomes (Supplemental Fig. S6A).

Next, to examine the genome-wide dynamic association between LMNB1 and repressive histone marks, we conducted principal component analyses (PCAs) on the ChIP-seq reads for LMNB1, H3K9me3, and H3K27me3 in growing and RIS cells. The profiles of H3K9me3 and H3K27me3 were highly distinct, and, consistent with results from our previous study (Chandra et al. 2012), the profile of each mark was globally unaltered during RIS. The DNA binding profile of LMNB1 was altered during RIS, and its orientation in the PCA was shifted away from the projection of $\mathrm{H} 3 \mathrm{~K} 9 \mathrm{me} 3$ to that of $\mathrm{H} 3 \mathrm{~K} 27 \mathrm{me} 3$, suggesting that the nature of the differential LMNB1 binding may be distinct between $\mathrm{H} 3 \mathrm{~K} 9 \mathrm{me} 3$ and H3K27me3 regions (Supplemental Fig. S6B). To confirm this, we performed PCAs for both the H3K9me3 and H3K27me3 reads in growing and RIS conditions and colored them differentially based on the nature of the LMNB1 alteration. The LMNB1-decreased and LMNB1-increased/ unchanged regions were biased toward the $\mathrm{H} 3 \mathrm{~K} 9 \mathrm{me} 3$ and H3K27me3 PCA projections, respectively (Fig. 2A). Consistently, H3K9me3 occupancy was relatively high in LMNB1-decreased regions (Grow > RIS), whereas LMNB1increased regions (Grow < RIS) tended to be more
H3K27me3-enriched (Supplemental Fig. S6C). These data indicate that LMNB1 binding is primarily reduced from H3K9me3-positive regions during RIS and that LMNB1 gain is more likely to occur in $\mathrm{H} 3 \mathrm{~K} 27 \mathrm{me} 3$-positive regions.

We next examined the alteration in LMNB1 binding in the $\mathrm{H} 3 \mathrm{~K} 9 \mathrm{me} 3$ and $\mathrm{H} 3 \mathrm{~K} 27 \mathrm{me} 3$-enriched regions in the context of LADs. It has been shown that LADs are enriched for $\mathrm{H} 3 \mathrm{~K} 27 \mathrm{me} 3$ at their edges, whereas $\mathrm{H} 3 \mathrm{~K} 9 \mathrm{me} 2$ occupies the whole LAD (Guelen et al. 2008). Our data agree with this and, in addition, we found a predominant enrichment of $\mathrm{H} 3 \mathrm{~K} 9 \mathrm{me} 3$ in the central region of LADs in growing conditions (Fig. 2B). Consistent with the PCA, the average reduction in LMNB1 was more prominent in the H3K9me3-enriched central regions than it was in the flanking H3K27me3 regions (Fig. 2C). A focused analysis of the LMNB1-increased regions ("RIS-up" + "RIS-only") revealed that gains in LMNB1 were closely associated with H3K27me3 regions (Fig. 2D). Thus, there is effectively an element of redistribution in LMNB1 together with a preferential reduction in LMNB1 from $\mathrm{H} 3 \mathrm{~K} 9 \mathrm{me} 3$ regions despite the global reduction of LMNB1 during RIS.

\section{Perinuclear heterochromatin is reduced during senescence}

In human diploid cells, an array of H3K9me3 foci are often observed in perinuclear regions, whereas H3K27me3 
A

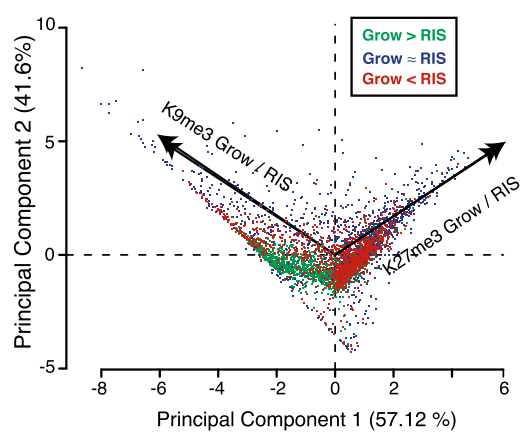

\section{B}

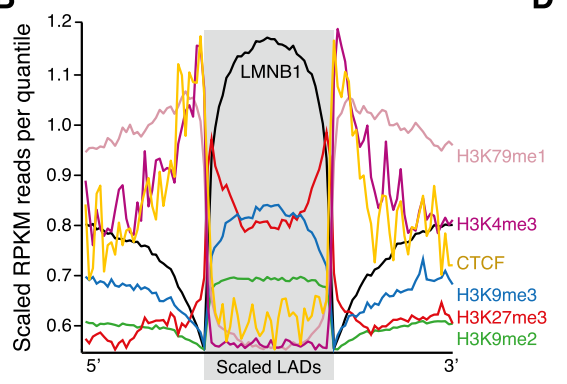

C

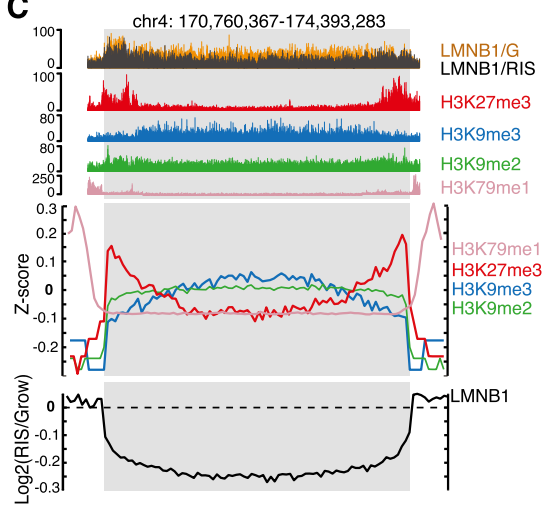

D

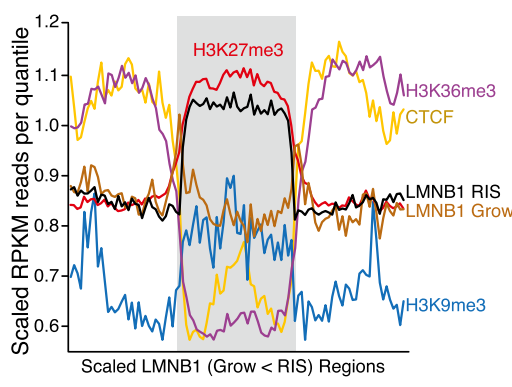

Figure 2. Preferential reduction of LMNB1 from H3K9me3 regions. (A) PCA of ChIP-seq data for $\mathrm{H} 3 \mathrm{~K} 9 \mathrm{me} 3$ and $\mathrm{H} 3 \mathrm{~K} 27 \mathrm{me} 3$ using a $500-\mathrm{kb}$ window size in growing and RIS conditions. LMNB1-positive segments in either condition are colored based on the classes defined in Figure 1D. (B) Profiles of the indicated chromatin features around LADs in growing cells. To align LADs, all LADs were segmented using a moving window of $1 \%$ of the domain size. LMNB1 and other ChIP-seq profiles were overlaid on the segmented windows using median reads per kilobase per million mapped (RPKM). (C, top) Genome browser representations of enrichment profiles of LMNB1 and indicated histone marks. The gray shading represents the LAD. (Middle) A standardized score ( $Z$-score) is used to show the signature of each histone mark overlapping the LAD. (Bottom) The log ratio of LMNB1 read counts between RIS and growing conditions around scaled LADs is shown. To avoid any contamination of the outside of scaled LADs with neighboring LADs, only short $(10-\mathrm{kb})$ leading and tailing regions were added. $(D)$ Profiles of histone marks overlapping aligned LMNB1-increased (Grow < RIS in Fig. 1D) regions (the gray shading) in the RIS condition. Domain alignment was per-

formed such that all domains were orientated $5^{\prime}$ to $3^{\prime}$; a distance equal to the domain width was added upstream of and downstream from the region, and the whole region was cut into equal 100 percentiles, where the LMNB1-enriched regions are now the mid-tertile of the data.

is localized to the interior of nuclei (Chandra et al. 2008, 2012). In addition, we showed recently that repressively marked chromatin is spatially repositioned during RIS to form SAHFs (Chandra et al. 2012). We found that the thickness of perinuclear electron-dense heterochromatin (by electron microscopy) and the number of H3K9me3 foci (by confocal microscopy) adjacent to the nuclear envelope were significantly diminished in RIS cells (Fig. $3 \mathrm{~A}, \mathrm{~B})$. Indeed, although some SAHFs contacted the nuclear envelop, the major part of the H3K9me3 core of SAHFs was located in the interior of the nucleus (Supplemental Movies S1, S2). The perinuclear heterochromatin reduction was also observed in replicative senescent IMR90 cells and RIS BJ cells (Supplemental Fig. S7A-D). In addition, oncogenic BRAF-induced senescent human melanocytes also exhibited SAHF formation as well as a reduction in perinuclear $\mathrm{H} 3 \mathrm{~K} 9 \mathrm{me} 3$ foci and LMNB1 levels, although the LMNB1 reduction in senescent melanocytes was less pronounced than in HDFs (Supplemental Fig. S7E-I). We hypothesized that the reduction of LMNB1, particularly from $\mathrm{H} 3 \mathrm{~K} 9 \mathrm{me} 3$ regions, during RIS facilitates the spatial redistribution of heterochromatin, providing a "pro-SAHF" nuclear environment. Although the reduction in perinuclear H3K9me3 foci was observed in both SAHF-positive and SAHF-negative RIS cells, it was more prominent in SAHF-positive cells (Fig. 3B). In addition, in single-cell analyses using laser-scanning cytometry (LSC) on IMR90 cells, we mapped the majority of SAHF-positive nuclei to the LMNB1-low RIS population (Fig. 3C). These data indicate that the reduction of both LMNB1 and perinuclear heterochromatin is closely correlated with SAHF formation.

To confirm that specific genomic regions do relocalize upon LMNB1 reduction during RIS, we used DNA fluorescence in situ hybridization (FISH). We chose seven bacterial artificial chromosome (BAC) probes mapped to genomic regions where LMNB1 became reduced to various degrees during RIS (Fig. 4A). These were mostly H3K9me3-enriched regions. Consistent with previous reports (Guelen et al. 2008; Haferkamp et al. 2009), we detected a correlation between the LMNB1 density associated with a particular DNA region and its perinuclear localization in combined samples from growing and RIS conditions. In addition, each probe tended to show a more perinuclear pattern in growing conditions relative to RIS (Fig. 4A). These results suggest that the global reduction in LMNB1 levels, particularly in $\mathrm{H} 3 \mathrm{~K} 9$ me3 regions, during RIS is correlated with the release of heterochromatin from the nuclear perimeter.

\section{Modulation of the global LMNB1 level affects SAHF formation}

To examine the direct consequences of LMNB1 reduction in normal HDFs, we generated shRNAs targeting LMNB1 using a miR30-based design (Fig. 4B; Silva et al. 2005). LMNB1 depletion in IMR90 and BJ cells resulted in a significant reduction in the number of perinuclear H3K9me3 foci, although it induced only a modest senes- 
A

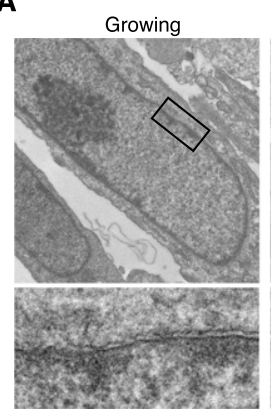

B

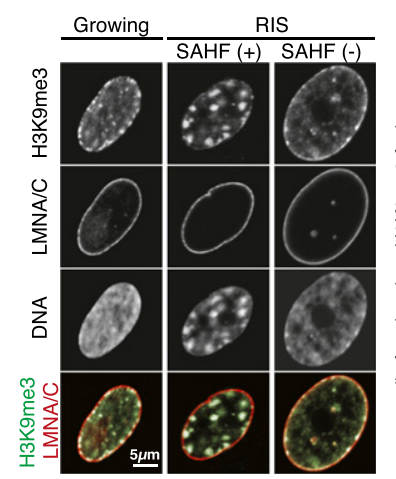

RIS
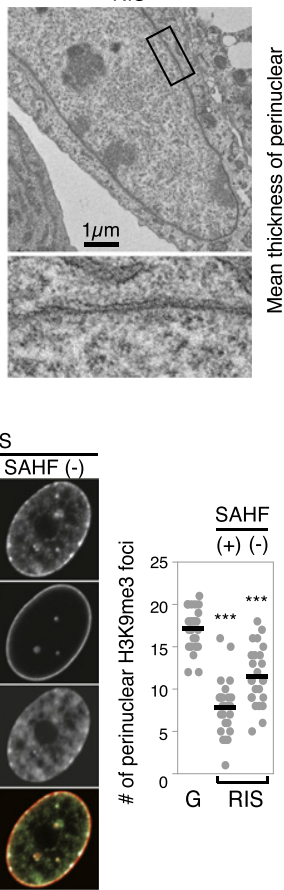

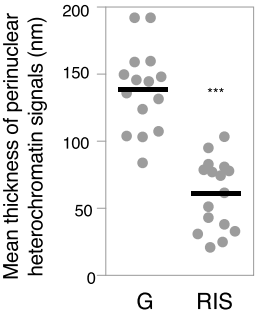

C

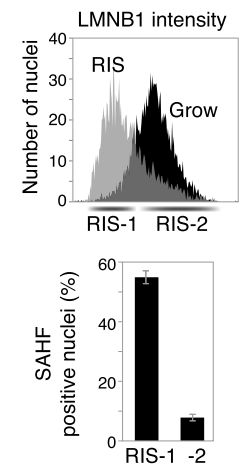

Figure 3. Reduction of perinuclear heterochromatin during senescence. (A) Representative electron micrographs of growing (G) and RIS IMR90 cells. Areas indicated by the rectangles are magnified. (Right) Thickness of high-electron density perinuclear areas was measured from the inner nuclear membrane using a superimposed grid and ImageJ. The data represent the average thickness of five to 17 points per nucleus. Black bars represent mean. Representative plot of two independent experiments is shown. $\left(^{\star \star \star}\right) P<0.001$. $(B)$ Confocal images of the equatorial section for the indicated antibodies in growing and RIS cells with $(+)$ or without $(-)$ obvious SAHFs. The number of H3K9me3 foci adjacent to the peripheral LMNA/C signals per nucleus $(n \geq 25)$ was counted. Black bars indicate mean. Representative plot of three independent experiments is shown. $\left({ }^{\star \star \star}\right) P<0.001 .(C)$ LMNB1 intensity per nucleus was quantified with a laser-scanning cytometer $(n>1000)$. (Top) Averaged histogram of three independent experiments is shown. (Bottom) RIS cells with low (RIS-1) and high (RIS-2) LMNB1 levels were gated, and SAHFs were assessed in each gated population.

cence phenotype (including SAHFs) (Fig. 4C; Supplemental Fig. S8A). We showed previously that the HMGA proteins are required for SAHF formation as architectural components (Narita et al. 2006). Thus, we hypothesized that the spatial removal of perinuclear heterochromatin together with the accumulation of these architectural components of SAHFs might cooperatively contribute to SAHF formation. Indeed, LMNB1 and HMGA2 protein levels were inversely correlated during the time course of RIS (Fig. 1A). To test this, we stably expressed sh-LMNB1 and/or HA-tagged HMGA proteins in IMR90 cells. Consistent with results from our previous study, overexpression of the HMGA proteins alone using a weak promoter showed a minimal effect, similar in extent to that of sh-LMNB1 alone (Fig. 4D; Narita et al. 2006). However, the coexpression of both sh-LMNB1 and HA-HMGA1 (or

HA-HMGA2) more efficiently induced SAHFs (Fig. 4D; Supplemental Fig. S8B). Similar results were also obtained using BJ cells (Supplemental Fig. S8C). We next examined whether ectopic expression of LMNB1 affects SAHF formation. The overall effect of LMNB1 overexpression on senescence has been controversial: LMNB1 overexpression was originally shown to increase the proliferation rate and delay the onset of senescence in HDFs (Shimi et al. 2011), but more recent studies suggested that it actually induces senescence (Barascu et al. 2012; Dreesen et al. 2013). When we ectopically expressed LMNB1 using the weak promoter, we managed to express it at a level comparable with endogenous LMNB1 in growing cells (Fig. 4E). In these conditions, while ectopic LMNB1 induced an irregular nuclear shape in both growing and RIS conditions, the impact on cell proliferation was marginal (Fig. 4E). Nevertheless, SAHF formation was partially, yet significantly, reduced by the ectopic expression of LMNB1 (Fig. 4E; Supplemental Fig. S8D). These data suggest that LMNB1 reduction during senescence induces the spatial repositioning of perinuclear heterochromatin and thus, together with architectural proteins, facilitates SAHF formation.

\section{De novo LMNB1 binding is correlated with gene repression}

It has been suggested that the association of genes with the nuclear lamina is involved in their silencing, but this repressive effect appears to depend on the genes and their chromatin context (Kind and Van Steensel 2010). Thus, we next examined whether there was any relationship between differential LMNB1 binding on genic regions and the differential expression of those genes during RIS. A genome-wide analysis failed to show any global correlation (Fig. 5A). This is consistent with a recent study showing that gene expression changes due to the double knockout of Lmnb1 and Lmnb2 in mouse trophectoderm and ESCs are not correlated with the binding of Lmnb1 to the promoters of those genes (Kim et al. 2011). Thus, our data suggest that expression of only a small subset of genes, if any, is correlated with the alteration of regional LMNB1 occupancy during RIS. To identify such genes, we took advantage of gene sets included in the regions defined in Figure 1D based on the differential binding of LMNB1 and performed a gene set enrichment analysis (GSEA) (Subramanian et al. 2005). This technique allowed us to test for a nonrandom distribution of an a priori defined subset of genes within a ranked expression data set. Genes within the RIS-only class were significantly down-regulated during RIS (Fig. 5B; Supplemental Table 2), while genes in all other classes failed to show significant association with the phenotype (Supplemental Fig. S9). High-scoring "leading-edge" genes (Subramanian et al. 2007) from the RIS-only class as well as other classes were subjected to a gene ontology (GO) analysis, revealing a distinct GO feature in the RIS-only class with prominent enrichment in cell cycle GO categories (Fig. 5C; see also Supplemental Fig. S5, where the examples overlap with leading-edge genes). In contrast, other classes mostly 
A
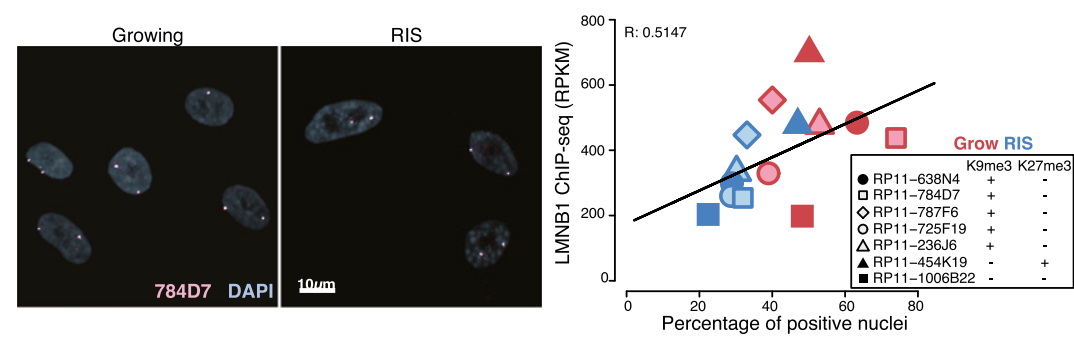

( $\geq 1$ perinuclear FISH signals)

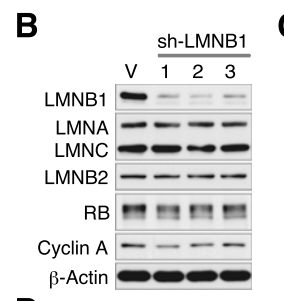

D

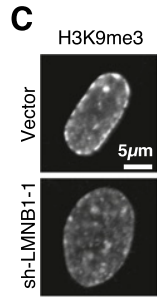

LMNB1
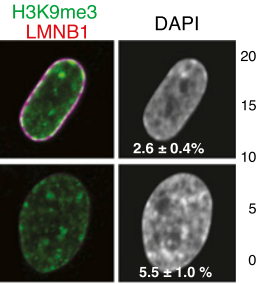

\# of perinuclear $\mathrm{H} 3 \mathrm{~K} 9 \mathrm{me} 3$ foci

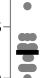

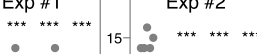

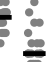

$\because 2 \frac{0}{10}$

$=\therefore x^{10-} \div$
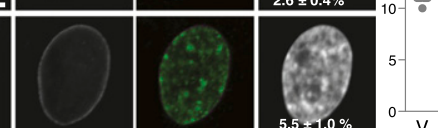

$\begin{array}{lllllllll}V & 1 & 2 & 3\end{array}$ V 1223

$\begin{array}{lllllll}\text { Sh-LMNB1-1 } & - & + & - & - & + & + \\ \text { HA:HMGA } & - & - & \text { A1 } & \text { A2 } & \text { A1 } & \text { A2 }\end{array}$

HA:HMGA - $-A 1$ A2 A1
LMNB1 -HA:HMGA2
HA:HMGA1

RB 브는

Cyclin A $-\ldots-\cdots$

$\beta$-Actin

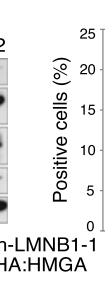

SAHFS

BrdU

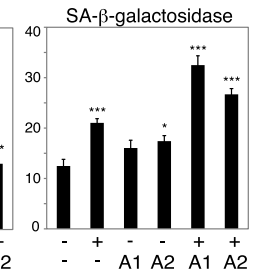

$\mathbf{E}$
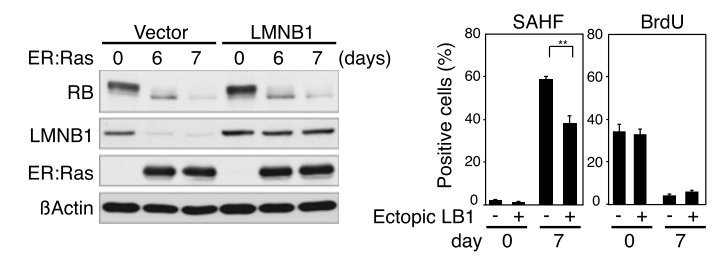

Figure 4. Correlation between LMNB1 reduction and intranuclear location of genomic regions. (A) Projections of confocal FISH images. (Left) FISH signals of the BAC clone 784D7 are shown. (Right) Mean percentage of cells that displayed at least one FISH signal at the nuclear periphery $(n=3)$ is plotted against mean RPKM of LMNB1 ChIP-seq within regions covered by each probe in each condition $(n=3)$. $(B)$ Western blot analysis for the indicated antibodies in IMR90 cells expressing three different shLMNB1. (C) Confocal images of LMNB1depleted cells stained with the indicated antibodies. (Left) Inset numbers represent percentage of cells with SAHFs (mean \pm SEM). (Right) The number of perinuclear H3K9me3 foci was assessed as in Figure 3B. Two independent experiments for three independent shRNAs are shown. $(D)$ Combination of LMNB1 depletion and ectopic expression of HMGA promotes SAHF formation and senescence. sh-LMNB1-1 or the control miR30 vector (-) and either HAHMGA1 (A1), HA-HMGA2 (A2), or the control vector $(-)$ were coexpressed in IMR90 cells. Cells were assessed for protein expression, SAHFs, BrdU incorporation, and SA$\beta$-galactosidase activity. Values represent mean \pm SEM from six experiments. $(E)$ Ectopic expression of LMNB1 inhibits SAHF formation. LMNB1 or control vector was retrovirally transduced to IMR90 cells expressing ER:Ras. Cells were assessed for protein expression, SAHFs, and BrdU incorporation at the indicated time points after ER:Ras induction. $\left(^{\star}\right) P<0.05 ;\left(^{\star \star}\right) P<0.01$; $\left({ }^{\star \star \star}\right) P<0.001$. share a "'membrane" signature, which we previously showed is strongly associated with RIS, suggesting that this correlation is likely to be nonspecific (Narita et al. 2011). Furthermore, the H3K27me 3 ratio between RIS and growing cells (log2 RIS/Grow) was elevated within the gene bodies of the leading-edge genes, whereas the same ratio for H3K36me3 dropped (Fig. 5D; Supplemental Fig. S10). These data suggest that the potential role of LMNB1 in gene repression is limited to a specific subset of genes representing a small fraction of the genome, wherein LMNB1 binding is paradoxically increased during RIS.

Finally, we performed additional DNA FISH experiments using probes that overlapped with the RIS-only region leading-edge genes, where $\mathrm{H} 3 \mathrm{~K} 27 \mathrm{me} 3$ was more prominent than H3K9me3 (Supplemental Fig. S11). Similar to the H3K27me3 immunolabeling pattern /Chandra et al. 2012), these regions were mostly located in the interior of the nucleus in both growing and RIS cells (Fig. 5E). In contrast to the first probe set, however, most RIS-only probes exhibited a shift toward the nuclear periphery, although the dynamic range was small (Fig. 5E; see Supplemental Fig. S11 for a better comparison between the two probe sets). These data further suggest that the regional gains in LMNB1 binding in part facilitate a perinuclear relocalization and thus may contribute to gene silencing.

\section{Discussion}

Increasing evidence has shown that LMNB1 global levels are altered during senescence, but the directionality of the change and its impact on the senescence phenotype are unclear (Shimi et al. 2011; Barascu et al. 2012; Freund et al. 2012; Dreesen et al. 2013). In this study, we confirmed that LMNB1 expression levels are down-regulated during senescence using human fibroblasts and melanocytes. At the same time, our data revealed that the alterations in LMNB1 binding to chromatin are uneven throughout the genome. This uneven redistribution is not random, since the major reductions in LMNB1 occur mostly in H3K9me3-enriched regions. In addition, in a small but substantial portion of the genome, particularly in H3K27me3-enriched regions, gains in LMNB1 binding occur during senescence. The global reduction and regional gains in LMNB1 binding are correlated with SAHF formation and gene repression, respectively; thus, it is possible that these two parallel processes collectively contribute to the senescence phenotype. 
A
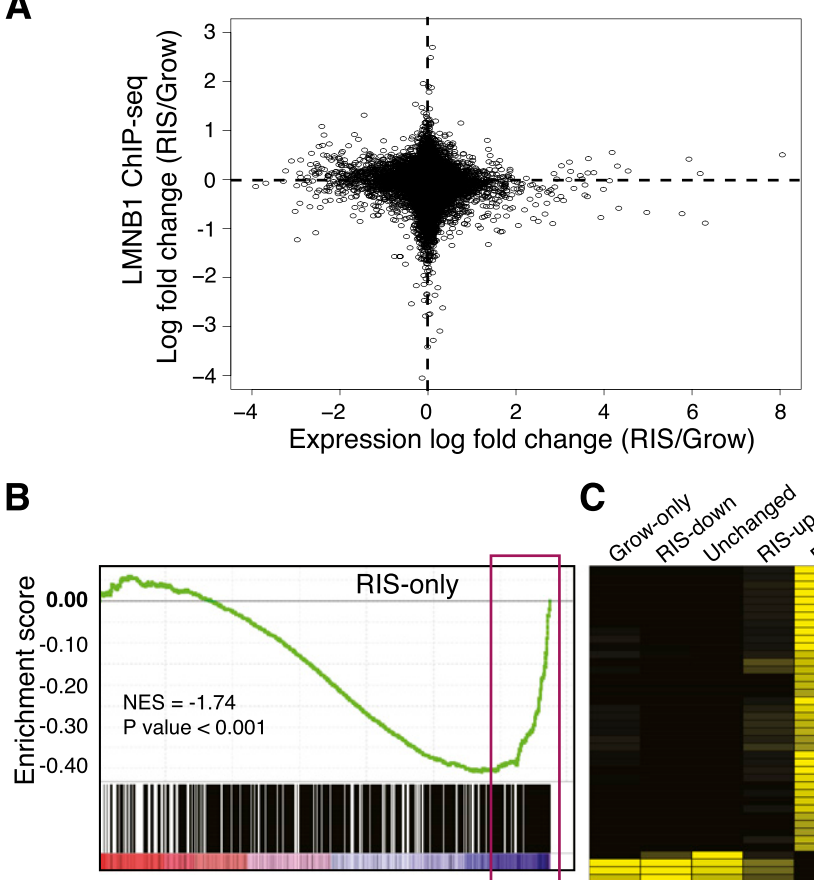

Rank in ordered dataset (Growing vs RIS)

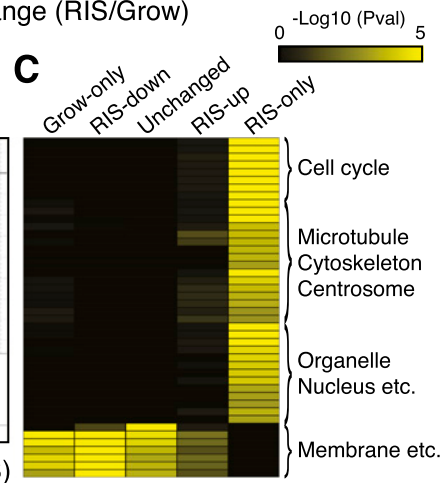

D

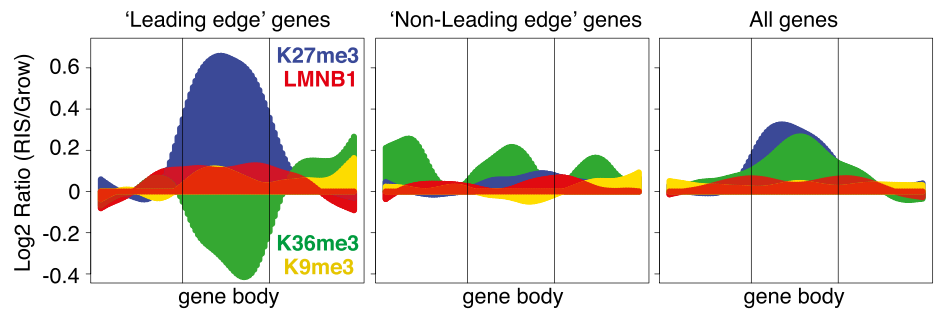

E

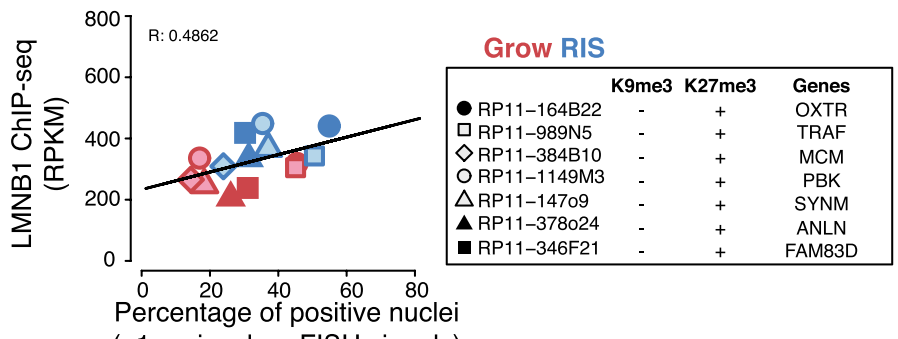

Figure 5. Gene repression in de novo LMNB1 regions in RIS cells. $(A)$ Scatter plot comparing $\log 2$ fold change of gene expression and LMNB1 ChIP-seq reads on the gene body during RIS. (B) GSEA profile of genes within the classes defined in Figure 1D. Genes are ranked into an ordered list based on differential expression between growing and RIS cells. Genes that are repressed during RIS are ranked toward the right (blue). The rectangle indicates the "leading-edge" subset of genes. $(C)$ Heat map showing GO analysis in genes in the leading-edge subsets from GSEA analyses of genes included in the indicated classes. $(D)$ Log2 ratios of normalized read counts for the indicated ChIP-seq data between RIS and growing conditions around scaled gene bodies associated with the RIS-only region are shown for the indicated gene sets. Gene bodies were aligned $5^{\prime}$ to $3^{\prime}$ according to transcription direction. $(B-D)$ Note that genes are included when at least $50 \%$ of gene bodies overlap with RIS-only regions. $(E)$ Localization of selected leading-edge genes by FISH in growing and RIS cells. Mean percentage of cells that displayed at least one FISH signal at the nuclear periphery $(n=3)$ is plotted against mean RPKM of LMNB1 ChIP-seq within regions covered by each probe in each condition $(n=3)$. See also Supplemental Figure S11.
High-order chromatin structure alteration is a complex and perhaps multimodular process. The "pro-SAHF" nuclear environment achieved through the preferential reduction of LMNB1 from $\mathrm{H} 3 \mathrm{~K} 9 \mathrm{me} 3$ regions appears to be independent of the architectural aspect of SAHF formation. Our findings therefore provide an additional element in the machinery of SAHF formation. Considering the modest effects of both the depletion and overexpression of LMNB1 on the process of SAHF formation, it is clear that other factors are involved in this process. It also remains to be elucidated how such an organized redistribution of LMNB1 during senescence is regulated. Interestingly, it has recently been shown that nuclear envelope proteins and their associated factors are involved in heterochromatin tethering to the nuclear periphery. For example, Lap2 $\beta$, a nuclear envelope protein, forms a complex with the transcription repressor cKrox (ThPOK) and the histone deacetylase HDAC3 and tethers LADs to the nuclear lamina in a sequence-dependent manner (Zullo et al. 2012). In addition, Lamin B receptor (LBR), another nuclear envelope protein, has been shown to contribute to tethering peripheral heterochromatin: The absence of both LBR and LMNA/C leads to the loss of peripheral heterochromatin (Solovei et al. 2013). LBR preferentially binds to B-type lamins as well as heterochromatin protein 1 (HP1), a H3K9me3-binding protein (Ye and Worman 1996). Although the dynamic status of these factors during senescence is not known, it is possible that, together with the global regulation of the LMNB1 level itself, modulation of more specific mecha- 
nisms involving genomic sequence- or histone markdependent chromatin tethering to the nuclear lamina contribute to the complex and uneven redistribution of LADs. Regardless of the nature of the mechanism, our results imply that the differential affinity of LMNB1 for regions with different genomic and chromatin features may itself be a mechanism that allows for the fine-tuning of LMNB1 functions. It has also been shown that LMNB1 defects affect interphase chromosomal positioning and gene expression (Malhas et al. 2007) and that the nuclear location of genes can change in a disease- and loci-specific manner during tumorigenesis (Meaburn and Misteli 2008; Meaburn et al. 2009). Thus, the regulation of the balance between alterations in LMNB1 on both global and regional levels might have wider implications for the development of disease.

\section{Materials and methods}

Antibodies

Antibodies used for Western blotting were as follows: LMNA/C (sc-7292, Santa Cruz Biotechnology), LMNB1 (ab16048, Abcam), Cyclin A (C4710, Sigma), p16 (sc-759, Santa Cruz Biotechnology), HMGA2 (sc-30223, Santa Cruz Biotechnology), H-Ras (OP23, Calbiochem), $\beta$-actin (A5441, Sigma), Rb (9309, Cell Signaling), HA (3724, Cell Signaling), and BRAF (F7, sc-5258, Santa Cruz Biotechnolgy).

\section{Cell culture and gene transfer}

IMR90 (female, embryonic lung-derived) and BJ (male, neonatal foreskin-derived) fibroblasts (American Type Culture Collection) were cultured in phenol-red-free DMEM with $10 \%$ FBS under $5 \%$ oxygen. The retroviral and lentiviral gene transfer was carried out as described (Narita et al. 2006) using the vectors detailed in the Supplemental Material. RIS was induced by retrovirusmediated expression of either H-Ras ${ }^{\mathrm{G} 12 \mathrm{~V}}$ or H-Ras ${ }^{\mathrm{G} 12 \mathrm{~V}}$ fused to the estrogen receptor (ER) ligand-binding domain (ER:Ras) (Young et al. 2009). Primary human melanocytes (Tissue Bank, Yale SPORE in Skin Cancer, Yale University) were grown in 254 medium, including a melanocyte growth supplement (Cascade Biologics/Life Technologies), under $5 \%$ oxygen. Senescence was induced by lentivirus-mediated expression of BRAF ${ }^{\mathrm{V} 600 \mathrm{E}}$ (Michaloglou et al. 2005).

Immunofluorescence, LSC, electron microscopy, and senescence assays

Immunofluorescence and senescence-associated $\beta$-galactosidase were performed as described (Narita et al. 2003). The primary antibodies used were H3K9me3 (07-523, Millipore), H3K27me3 (CMA323) (Hayashi-Takanaka et al. 2011), LMNA/C (sc-7292, Santa Cruz Biotechnology), LMNB1 (ab16048, Abcam; sc-6216, Santa Cruz Biotechnology), HA (H9658, Sigma), and BrdU (555627, Becton Dickinson). For quantitative imaging, LSC (Compucyte iCys) was used. Electron microscopy was performed as reported (Motskin et al. 2011).

\section{ChIP-seq}

ChIP was performed as described (Chandra et al. 2012). For LMNB1 and control IgG ChIP-seq, three independent biological replicates were performed with matched inputs for each con- dition using a LMNB1 antibody (ab16048, Abcam) and then anti-rabbit IgG-conjugated Dynabeads (112.04D, Invitrogen). All ChIP-seq data from this study have been submitted to the Gene Expression Omnibus database (GSE49341). All other ChIP-seq data came from our previous study and were obtained using the same conditions (Chandra et al. 2012), except for H3K79me1 (Hawkins et al. 2010). CTCF-binding regions were obtained from published data (Kim et al. 2007). For detailed ChIP-seq data analyses, see the Supplemental Material.

\section{Expression microarray}

The gene expression data sets on HumanHT-12 version 3.0 Expression BeadChip (Illumina) arrays were those published previously (Chandra et al. 2012). For detailed data analyses, see the Supplemental Material.

\section{DNA-FISH}

DNA-FISH was performed as described elsewhere (Chandra et al. 2012).

\section{Acknowledgments}

We thank members of the Narita laboratory for helpful discussions; L. Blackburn for editing; CI core Genomics, Bioinformatics, and Microscopy (H. Zecchini and A. Schreiner) facilities for technical support; J. Skepper for electron microscopy; and D. Peeper, P. Adams, and M. Soengas for reagents and technical advice. This work was supported by the University of Cambridge; Cancer Research UK; Hutchison Whampoa; the Human Frontier Science Program (M.N. and R.S.); Cancer Research UK consortium grant, Senectus Therapeutics (M.N., D.C.B., and M.S.); and fellowships from the Uehara Memorial Foundation (M.S.) and the Japan Society for the Promotion of Science (K.T.).

\section{References}

Barascu A, Le Chalony C, Pennarun GEL, Genet D, Imam N, Lopez B, Bertrand P. 2012. Oxidative stress induces an ATM-independent senescence pathway through p38 MAPKmediated lamin B1 accumulation. EMBO I 31: 1080-1094.

Burke B, Stewart CL. 2012. The nuclear lamins: Flexibility in function. Nat Rev Mol Cell Biol 14: 13-24.

Chan HM, Narita M, Lowe SW, Livingston DM. 2005. The p400 E1A-associated protein is a novel component of the p53 $\rightarrow$ p21 senescence pathway. Genes Dev 19: 196-201.

Chandra T, Narita M. 2013. High-order chromatin structure and the epigenome in SAHFs. Nucleus 4: 23-28.

Chandra T, Kirschner K, Thuret J-Y, Pope BD, Ryba T, Newman S, Ahmed K, Samarajiwa SA, Salama R, Carroll T, et al. 2012. Independence of repressive histone marks and chromatin compaction during senescent heterochromatic layer formation. Mol Cell 47: 203-214.

Collado M, Serrano M. 2006. The power and the promise of oncogene-induced senescence markers. Nat Rev Cancer 6: 472-476.

Dechat T, Adam SA, Taimen P, Shimi T, Goldman RD. 2010. Nuclear lamins. Cold Spring Harb Perspect Biol 2: a000547.

Dittmer TA, Misteli T. 2011. The lamin protein family. Genome Biol 12: 222.

Dreesen O, Chojnowski A, Ong PF, Zhao TY, Common JE, Lunny D, Lane EB, Lee SJ, Vardy LA, Stewart CL, et al. 2013. Lamin B1 fluctuations have differential effects on cellular proliferation and senescence. J Cell Biol 200: 605617. 
Freund A, Laberge RM, Demaria M, Campisi J. 2012. Lamin B1 loss is a senescence-associated biomarker. Mol Biol Cell 23: 2066-2075.

Funayama R, Saito M, Tanobe H, Ishikawa F. 2006. Loss of linker histone $\mathrm{H} 1$ in cellular senescence. I Cell Biol 175: 869-880.

Guelen L, Pagie L, Brasset E, Meuleman W, Faza MB, Talhout W, Eussen BH, De Klein A, Wessels L, De Laat W, et al. 2008 . Domain organization of human chromosomes revealed by mapping of nuclear lamina interactions. Nature 453: 948-951.

Haferkamp S, Tran SL, Becker TM, Scurr LL, Kefford RF, Rizos H. 2009. The relative contributions of the p53 and $\mathrm{pRb}$ pathways in oncogene-induced melanocyte senescence. $A g$ ing (Albany, NY Online) 1: 542-556.

Hawkins RD, Hon GC, Lee LK, Ngo Q, Lister R, Pelizzola M, Edsall LE, Kuan S, Luu Y, Klugman S, et al. 2010. Distinct epigenomic landscapes of pluripotent and lineage-committed human cells. Cell Stem Cell 6: 479-491.

Hayashi-Takanaka Y, Yamagata K, Wakayama T, Stasevich TJ, Kainuma T, Tsurimoto T, Tachibana M, Shinkai Y, Kurumizaka H, Nozaki N, et al. 2011. Tracking epigenetic histone modifications in single cells using Fab-based live endogenous modification labeling. Nucleic Acids Res 39: 6475-6488.

Kim TH, Abdullaev ZK, Smith AD, Ching KA, Loukinov DI, Green RD, Zhang MQ, Lobanenkov VV, Ren B. 2007. Analysis of the vertebrate insulator protein CTCF-binding sites in the human genome. Cell 128: 1231-1245.

Kim Y, Sharov AA, McDole K, Cheng M, Hao H, Fan CM, Gaiano N, Ko MSH, Zheng Y. 2011. Mouse B-type lamins are required for proper organogenesis but not by embryonic stem cells. Science 334: 1706-1710.

Kimura H, Hayashi-Takanaka Y, Goto Y, Takizawa N, Nozaki N. 2008. The organization of histone H3 modifications as revealed by a panel of specific monoclonal antibodies. Cell Struct Funct 33: 61-73.

Kind J, Van Steensel B. 2010. Genome-nuclear lamina interactions and gene regulation. Curr Opin Cell Biol 22: 320-325.

Malhas A, Lee CF, Sanders R, Saunders NJ, Vaux DJ. 2007. Defects in lamin B1 expression or processing affect interphase chromosome position and gene expression. J Cell Biol 176: $593-603$.

McClintock D, Gordon LB, Djabali K. 2006. Hutchinson-Gilford progeria mutant lamin A primarily targets human vascular cells as detected by an anti-Lamin A G608G antibody. Proc Natl Acad Sci 103: 2154-2159.

Meaburn KJ, Misteli T. 2008. Locus-specific and activityindependent gene repositioning during early tumorigenesis. I Cell Biol 180: 39-50.

Meaburn KJ, Gudla PR, Khan S, Lockett SJ, Misteli T. 2009. Disease-specific gene repositioning in breast cancer. J Cell Biol 187: 801-812.

Michaloglou C, Vredeveld LCW, Soengas MS, Denoyelle C, Kuilman T, van der Horst CMAM, Majoor DM, Shay JW, Mooi WJ, Peeper DS. 2005. BRAFE600-associated senescencelike cell cycle arrest of human naevi. Nature 436: 720-724.

Motskin M, Müller KH, Genoud C, Monteith AG, Skepper JN. 2011. The sequestration of hydroxyapatite nanoparticles by human monocyte-macrophages in a compartment that allows free diffusion with the extracellular environment. Biomaterials 32: 9470-9482.

Narita M, Nũnez S, Heard E, Narita M, Lin AW, Hearn SA, Spector DL, Hannon GJ, Lowe SW. 2003. Rb-mediated heterochromatin formation and silencing of E2F target genes during cellular senescence. Cell 113: 703-716.

Narita M, Narita M, Krizhanovsky V, Nũnez S, Chicas A, Hearn SA, Myers MP, Lowe SW. 2006. A novel role for high-mobility group a proteins in cellular senescence and heterochromatin formation. Cell 126: 503-514.

Narita M, Young ARJ, Arakawa S, Samarajiwa SA, Nakashima T, Yoshida S, Hong S, Berry LS, Reichelt S, Ferreira M, et al. 2011. Spatial coupling of mTOR and autophagy augments secretory phenotypes. Science 332: 966-970.

Peric-Hupkes D, Meuleman W, Pagie L, Bruggeman SWM, Solovei I, Brugman W, Gräf S, Flicek P, Kerkhoven RM, Van Lohuizen M, et al. 2010. Molecular maps of the reorganization of genome-nuclear lamina interactions during differentiation. Mol Cell 38: 603-613.

Pickersgill H, Kalverda B, de Wit E, Talhout W, Fornerod M, Van Steensel B. 2006. Characterization of the Drosophila melanogaster genome at the nuclear lamina. Nat Genet 38: 10051014.

Serrano M, Lin AW, McCurrach ME, Beach D, Lowe SW. 1997. Oncogenic ras provokes premature cell senescence associated with accumulation of p53 and p16INK4a. Cell 88: 593-602.

Sharpless NE, DePinho RA. 2007. How stem cells age and why this makes us grow old. Nat Rev Mol Cell Biol 8: 703-713.

Shelton DN, Chang E, Whittier PS, Choi D, Funk WD. 1999. Microarray analysis of replicative senescence. Curr Biol 9: 939-945.

Shimi T, Butin-Israeli V, Adam SA, Hamanaka RB, Goldman AE, Lucas CA, Shumaker DK, Kosak ST, Chandel NS, Goldman RD. 2011. The role of nuclear lamin B1 in cell proliferation and senescence. Genes Dev 25: 2579-2593.

Silva JM, Li MZ, Chang K, Ge W, Golding MC, Rickles RJ, Siolas D, Hu G, Paddison PJ, Schlabach MR, et al. 2005. Secondgeneration shRNA libraries covering the mouse and human genomes. Nat Genet 37: 1281-1288.

Solovei I, Wang AS, Thanisch K, Schmidt CS, Krebs S, Zwerger M, Cohen TV, Devys D, Foisner R, Peichl L, et al. 2013. LBR and lamin $\mathrm{A} / \mathrm{C}$ sequentially tether peripheral heterochromatin and inversely regulate differentiation. Cell 152: 584598.

Subramanian A, Tamayo P, Mootha VK, Mukherjee S, Ebert BL, Gillette MA, Paulovich A, Pomeroy SL, Golub TR, Lander ES, et al. 2005. Gene set enrichment analysis: A knowledgebased approach for interpreting genome-wide expression profiles. Proc Natl Acad Sci 102: 15545-15550.

Subramanian A, Kuehn H, Gould J, Tamayo P, Mesirov JP. 2007. GSEA-P: A desktop application for gene set enrichment analysis. Bioinformatics 23: 3251-3253.

Ye Q, Worman HJ. 1996. Interaction between an integral protein of the nuclear envelope inner membrane and human chromodomain proteins homologous to Drosophila HP1. I Biol Chem 271: 14653-14656.

Ye X, Zerlanko B, Kennedy A, Banumathy G, Zhang R, Adams PD. 2007. Downregulation of Wnt signaling is a trigger for formation of facultative heterochromatin and onset of cell senescence in primary human cells. Mol Cell 27: 183-196.

Young ARJ, Narita M, Ferreira M, Kirschner K, Sadaie M, Darot JFJ, Tavaré S, Arakawa S, Shimizu S, Watt FM, et al. 2009. Autophagy mediates the mitotic senescence transition. Genes Dev 23: $798-803$.

Zhang R, Poustovoitov MV, Ye X, Santos HA, Chen W, Daganzo SM, Erzberger JP, Serebriiskii IG, Canutescu AA, Dunbrack $\mathrm{RL}$, et al. 2005. Formation of macroH2A-containing senescence-associated heterochromatin foci and senescence driven by ASF1a and HIRA. Dev Cell 8: 19-30.

Zullo JM, Demarco IA, Piqué-Regi R, Gaffney DJ, Epstein CB, Spooner CJ, Luperchio TR, Bernstein BE, Pritchard JK, Reddy $\mathrm{KL}$, et al. 2012. DNA sequence-dependent compartmentalization and silencing of chromatin at the nuclear lamina. Cell 149: $1474-1487$. 


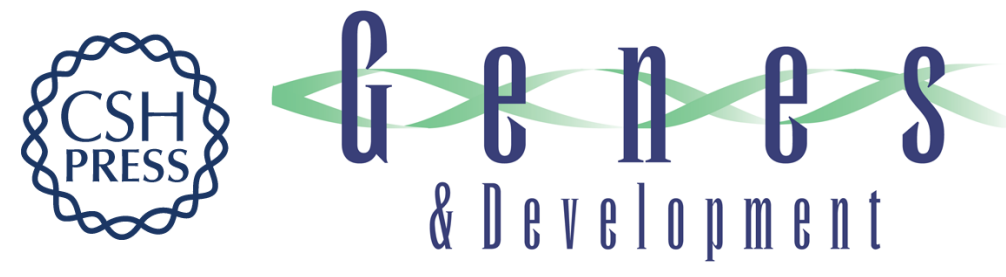

\section{Redistribution of the Lamin B1 genomic binding profile affects rearrangement of heterochromatic domains and SAHF formation during senescence}

Mahito Sadaie, Rafik Salama, Thomas Carroll, et al.

Genes Dev. 2013, 27:

Access the most recent version at doi:10.1101/gad.217281.113

Supplemental http://genesdev.cshlp.org/content/suppl/2013/08/20/27.16.1800.DC1

Material

References This article cites 43 articles, 15 of which can be accessed free at: http://genesdev.cshlp.org/content/27/16/1800.full.html\#ref-list-1

Creative This article, published in Genes \& Development, is available under a Creative Commons

Commons License (Attribution-NonCommercial 3.0 Unported), as described at

License http://creativecommons.org/licenses/by-nc/3.0/.

Email Alerting Receive free email alerts when new articles cite this article - sign up in the box at the top Service right corner of the article or click here.

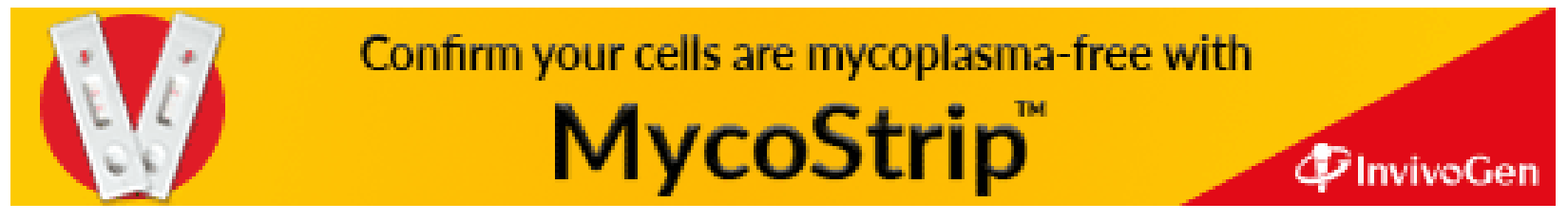

